\section{Diagnosis of Chlamydophila felis by conjunctival cytology in shelter cats}

\section{David Mills}

RSPCA Putney Animal Hospital, London, UK

\section{BACKGROUND}

Chlamydophila felis (C.felis) has a prevalence of $7-56 \%$ in cats with upper respiratory signs and conjunctivitis, and is commonly suspected in cats with upper respiratory disease (FURD) presented at shelters. It requires lengthy and costly treatment for resolution. Accurate diagnosis is imperative and a test with high sensitivity minimises in-shelter infection transmission from false negatives. Given the considerable overlap with other causes of FURD, clinical signs alone are unreliable. The current gold standard of external laboratory PCR is expensive and confers a time delay of 48-72 hours. Cytology of conjunctival swabs can be used to identify chlamydial inclusion bodies in conjunctival epithelial cells in conjunction with an inflammatory reaction. It is rapid, requires no special equipment, and is within the competence of general practitioners. However its sensitivity and specificity have not been widely evaluated.

\section{METHODS AND MATERIALS}

In a prospective single-blinded study, conjunctival swabs were taken from 36 stray cats suspected of being infected with C.felis presented to a RSPCA hospital in London over a
6 month period. Sterile cotton buds, 2 per cat, were used to collect samples by rubbing the ventral conjunctiva of the same eye following local anaesthetic administration. Slides for cytological evaluation were prepared from one swab and the other was submitted for external PCR analysis. Swabs were taken by one practitioner and assessed by another, who was blinded to the case in question. Slides were stained with commercial Romanowsky in-house stains and evaluated under 100x magnification. Practitioners had received brief prior training in cytological diagnosis of $C$.felis.

\section{RESULTS}

28/36 (78\%) of all cats were PCR-positive. 26/28 PCR-positive cats were correctly diagnosed cytologically. 3/8 PCRnegative cats were incorrectly diagnosed cytologically as being C.felis infected. Conjunctival cytology had a sensitivity of $93 \%$ and specificity of $63 \%$ for diagnosis of C.felis. No influence of clinical signs on cytological accuracy were seen. All cats were estimated as being less than 18 months of age.

\section{DISCUSSION/CONCLUSION}

In this study and population of cats, conjunctival cytology represented a cheap, rapid and sensitive test for $C$.felis. The sensitivity of cytology is reported to drop the longer the cat has been infected, which may explain the false negatives reported. False positives are possible from practitioner inexperience, or misinterpretation of melanin granules or bacteria. Cytology may represent a useful, inexpensive tool in a multi-dimensional shelter protocol for targeted therapy of cats presenting with FURD. 